\title{
Nitrogen-induced hardening in an austenitic CrFeMnNi high- entropy alloy (HEA)
}

\author{
Mathieu Traversier ${ }^{1}$, Pierre Mestre-Rinn ${ }^{1}$, Nathalie Peillon ${ }^{1}$, Emmanuel Rigal $^{2}$, Xavier Boulnat $^{3}$, \\ Franck Tancret ${ }^{4}$, Jean Dhers ${ }^{5}$ and Anna Fraczkiewicz ${ }^{1}$ \\ ${ }^{1 .}$ MINES Saint-Etienne, Université de Lyon, CNRS, UMR 5307 LGF, Centre SMS, 42023 Saint-Etienne, \\ FRANCE \\ 2. CEA/LITEN, F-38054 Grenoble, FRANCE \\ ${ }^{3}$ Université de Lyon, INSA Lyon, MATEIS UMR CNRS 5510, 69621 Villeurbanne Cedex, FRANCE. \\ ${ }^{4}$ Université de Nantes, CNRS, Institut des Matériaux Jean Rouxel, IMN, 44000 Nantes, France \\ ${ }^{5}$ FRAMATOME, Lyon, FRANCE \\ Corresponding Author: mathieu.traversier@emse.fr
}

\section{Abstract}

Nitrogen is a well-known gamma-stabiliser in austenitic steels, also responsible for significant solid solution hardening of these materials. Yet, only few papers have studied its impact on austenitic high-entropy alloy (HEA) matrixes. This study focuses on a cobalt-free, non equimolar $\mathrm{CrFeMnNi}$ HEA doped with nitrogen. A series of alloys was cast under a nitriding atmosphere to promote nitrogen absorption into the liquid alloy. Study of as-cast alloys has shown nitrogen presence in solid solution up to $0.3 \mathrm{wt}$. \% (1.2 at. \%). Over the whole range of compositions, a linear increase of hardness $(134 \mathrm{HV} / \mathrm{wt}$. \% of $\mathrm{N})$ was measured as well as an expansion of the lattice parameter of $\Delta \mathrm{a} / \mathrm{a}=1.01 / \mathrm{wt} . \% \mathrm{~N}$ due to nitrogen addition in the interstitial sites of the lattice. Tests on forged and annealed samples showed that the increase of hardness with nitrogen addition is higher than in as-cast state (210 HV / wt. \% of N) surely due to presence of other strengthening mechanisms. Tensile tests confirmed that the presence of dissolved nitrogen increases yield strength and ultimate strength and enhances strain-hardening, without any modification of ductility.

\section{Keywords}

High entropy alloys (HEA); Nitrogen alloying; Solid solution; Microstructure; Tensile properties

\section{1- Introduction}

High-entropy alloys (HEAs) were initially described as alloys containing at least five metallic elements, each one present in an atomic proportion between 5 and 35\%. The first HEA to be discovered was an equimolar CoCrFeMnNi, often denominated today as the Cantor alloy [1]. During the fifteen years of existence of HEAs, the concepts have strongly evolved. The four "core effects" proposed by Yeh [2] (high distortion of the lattice due to the different atoms sizes; sluggish diffusion; high entropy effect to stabilise the solid solution; the "cocktail effect") to describe the specific behaviour of HEAs have been partially questioned, as shown, for instance, by Miracle and Senkov [3] in a recent review. Especially, the entropy effect is far from being at the origin of the specific behaviour of HEAs, as similar properties have been identified in an equimolar ternary CoCrNi alloy [4].

Nowadays, the Cantor alloy still remains the most studied, but is mainly considered as a model alloy to understand specific behaviours of HEAs. This alloy presents a stable face-centered cubic (FCC) structure, amagnetic at all temperatures. Contrarily to some austenitic stainless steels, it does not exhibit any martensitic transformation, even under mechanical stresses. It shows a quite unusual simultaneous increase of both yield strength and ductility with decreasing temperature [5]. Moreover, its impact resistance is high, especially at cryogenic temperatures. These effects are attributed to the decrease of the stacking fault energy (SFE) with temperature [5], [6]. In these conditions, twin formation is promoted; their presence acts as dislocation barriers creating a 
"dynamic Hall-Petch effect" [7]. This effect leads to the increase of the strain-hardening rate during deformation, expanding the amount of elongation before necking appears, thus improving the ductility of the alloy. However, as other classical FCC structures, the Cantor alloy suffers from a common weakness: a lack of strength. This is why various ways of increasing its strength, like precipitation hardening [8], [9] or the formation of "duplex structures" due to a body-centered cubic (BCC) phase precipitation [10] are investigated. For the same purpose, because the potential extent of substitutional solid solution hardening seems limited in FCC HEAs [3], interstitial alloying has received a lot of interest recently. Several studies showed that carbon additions in HEAs can greatly improve yield strength while having little, no or even positive impact on ductility [11], [12]. Li et al. showed that a $\mathrm{Fe}_{50} \mathrm{Mn}_{30} \mathrm{Co}_{10} \mathrm{Cr}_{10}$ grade doped with $0.1 \mathrm{wt}$. \% of carbon $(0.5$ at. \%) exhibits both twinning and transformation induced plasticity (TWIP and TRIP effects), leading to a very high combination of strength and ductility [13]. Nitrogen, another interstitial element commonly present in stainless steels, could also create promising properties.

Actually, among FCC stabilizing elements used in conventional steels, nitrogen is known to be the most powerful one and was used as a substitution for $\mathrm{Ni}$ when its price significantly increased, leading to a new type of alloys rich in $\mathrm{Cr}$ and $\mathrm{Mn}$ : High Nitrogen Stainless Steels. Moreover, nitrogen has other beneficial effects: its addition is efficient to increase the yield strength and ultimate tensile strength and to promote strain-hardening, improves fatigue properties and resistance to localized corrosion, as well as helps grain size refinement [14], [15], [16]. Finally, nitrogen is also widely used in industry to increase the mechanical properties of surfaces through nitriding. Promising properties of high entropy nitride films were recently reported in the literature [17], [18], [19].

Similarly, nitrogen additions as an alloying element in bulk HEA has already been reported in several studies. While in most of them, nitrogen is introduced in the alloy during melting thanks to a high gas pressure [20], [21], [22], [23], it can also be introduced into the molten alloy in the form of nitride like $\mathrm{FeCrN}_{2}$ [24], [25], [26], [27] or during mechanical alloying with nitride powders [28], [29]. Han et al. [23], have shown the efficiency of nitrogen to increase the mechanical resistance of a cold-rolled Cantor alloy doped with 0.52 at.\% of $\mathrm{N}(0.13$ wt. \%): these effects have been attributed to the increase of lattice friction, planar dislocation slip and twinning activation. Similar effects have been observed by Jodi et al. [20], [21] and Moravcik et al. [21] on the ternary alloy CoCrNi containing 0.95 at. $\% \mathrm{~N}(0.24$ wt. \%) and 0.47 at. $\% \mathrm{~N}(0.12$ wt. \%) respectively. Moreover, these authors suggest that the presence of intergranular $\mathrm{Cr}_{2} \mathrm{~N}$ precipitates might participate to Zener pinning and cause the reduction of grain size during processing. Klimova et al. studied the behaviour of a Cantor alloy doped with nitrogen up to 2 at. $\% \mathrm{~N}(0.49 \mathrm{wt} . \% \mathrm{~N})$ at cryogenic temperature [26]. Nitrogen's strengthening through solid solution is even more important than at room temperature making nitrogen-doped HEAs promising solutions for cryogenic applications. It should however be noted that most of those studied alloys (Cantor alloy and $\mathrm{CoCrNi}$ ) have a large amount of Co which is known to reduce nitrogen solubility in stainless steels [16]. While no severe nitride precipitation was reported, surely due to the slow precipitation kinetics of $\mathrm{Cr}_{2} \mathrm{~N}$ in austenitic matrixes [30], Co-rich HEAs may not be the best candidates for nitrogen alloying.

In the present work, we aim to study the effect of nitrogen addition on a HEA from the CrFeMnNi family, the so called Y3 grade $\left(\mathrm{Cr}_{15} \mathrm{Fe}_{46} \mathrm{Mn}_{17} \mathrm{Ni}_{22}\right.$, at. \%), designed and developed recently [31]. This alloy, similar to the Cantor alloy, possesses a single phased FCC structure and keeps the main assets of its prototype: increased cryogenic mechanical resistance, absence of martensitic transformation and paramagnetic behaviour over the whole range of temperatures. Moreover, the perk of being cobalt-free decreases its cost and makes it a good candidate for some specific applications, like in the nuclear industry for instance, due to its low activation under irradiation. In the present study, nitrogen additions to Y3 alloy will be analysed and quantified from structural, microstructural and mechanical points of view. 


\section{2- Experimental}

To evaluate the effect of nitrogen on the alloy, three nitrogen concentrations were targeted: $0.1 / 0.17$ and 0.3 wt. \% $\mathrm{N}(0.4 / 0.69$ and 1.2 at. \% respectively) and were compared to the nitrogen-free alloy. Different nitrogen-doped alloys (300 g ingots) based on the "Y3" composition have been elaborated by cold crucible melting starting from high purity metals. Nitrogen was afterwards introduced in the liquid alloy through exposure to gaseous $\mathrm{N}_{2}$ under controlled pressure and time. The chemical composition of as-cast alloys was checked by Inductively Coupled Plasma (ICP) for metallic elements and infrared absorption of combustion gases (LECO, TC-436) for C, N, $\mathrm{S}, \mathrm{O}$. The results of chemical analysis showed that the nitrogen concentration is close to the targeted one (Table 1).

Table 1. Chemical composition of studied alloys as measured by ICP and LECO methods. Compositions are given in weight percent or weight ppm, for metallic elements and impurities, respectively. The used abbreviations corresponds to the material's state: AC: as-cast; FA: forgedannealed

\begin{tabular}{ccccccccc}
\hline & $\mathrm{Fe}$ & $\begin{array}{c}\mathrm{Cr} \\
(\mathrm{wt} \%)\end{array}$ & $\begin{array}{c}\mathrm{Ni} \\
(\mathrm{wt} \%)\end{array}$ & $\begin{array}{c}\mathrm{Mn} \\
(\mathrm{wt} \%)\end{array}$ & $\begin{array}{c}\mathrm{N} \\
(\mathrm{wt} \mathrm{ppm})\end{array}$ & $\begin{array}{c}\mathrm{O} \\
(\mathrm{wt} \mathrm{ppm})\end{array}$ & $\begin{array}{c}\mathrm{S} \\
(\text { wt ppm })\end{array}$ & $\begin{array}{c}\mathrm{C} \\
\text { (wt ppm })\end{array}$ \\
\hline Y3 Target & Base & 14.0 & 23.0 & 17.0 & $/$ & $/$ & $/$ & $/$ \\
\hline Y3-0-AC & Base & 14.0 & 23.6 & 16.9 & $\mathbf{8}$ & 15 & 44 & 11 \\
\hline Y3-0.17-AC & Base & 14 & 23.3 & 17.1 & $\mathbf{1 7 5 0}$ & 13 & 45 & 19 \\
\hline Y3-0.3-AC & Base & 13.8 & 22.9 & 16.7 & $\mathbf{2 9 0 0}$ & 1 & 47 & 7 \\
\hline Y3-0-FA & Base & 14.0 & 23.6 & 16.9 & $\mathbf{1 0}$ & 10 & 44 & 15 \\
\hline Y3-0.1-FA & Base & 14.4 & 22.7 & 16.9 & $\mathbf{1 1 0 0}$ & 30 & 49 & 14 \\
\hline Y3-0.17-FA & Base & 14 & 23.3 & 17.1 & $\mathbf{1 7 5 0}$ & 4 & 48 & 20 \\
\hline Y3-0.3-FA & Base & 14.5 & 22.9 & 17.5 & $\mathbf{2 7 8 0}$ & 20 & 42 & 7 \\
\hline
\end{tabular}

For all alloys, the content of oxygen and carbon, in agreement with our expectations, is below $30 \mathrm{wt}$. ppm. The higher amount of $\mathrm{S}$, about $50 \mathrm{wt}$. ppm, is due to a poorer quality of manganese used for this elaboration and may result in the formation of scarce MnS inclusions. Yet, its content remains constant for all the series of alloys. The effect of sulphur will thus be neglected.

As-cast alloys were also transformed by forging at approximately $1000^{\circ} \mathrm{C}$ to produce $14 \mathrm{~mm}$ diameter bars. After forging, samples were annealed at $1000^{\circ} \mathrm{C}$ for 2 hours and water quenched. In the following, samples will be referred to following this format: Y3-A-B with A being the nitrogen concentration (i.e. $0 / 0.1 / 0.17$ or $0.3 \mathrm{wt} . \% \mathrm{~N}$ ) and $\mathrm{B}$ the state of the sample (AC for AsCast or FA for Forged-Annealed). For instance, the Y3-0.17-AC sample contains 0.17 wt. \% N and has been studied in its as-cast state. One can note in Table 1 that the nitrogen concentration of Y30.1 and Y3-0.3 grades differs between the AC state and the FA state $(0.09$ vs 0.11 wt. \% N for Y30.1 and 0.29 vs 0.28 wt. $\% \mathrm{~N}$ for Y3-0.3). They are actually different castings. Still, as the difference in nitrogen concentration is quite low between the two states and near the targeted value, their behaviour should remain similar and no distinction will be made later on.

For Scanning Electron Microscope (SEM) observations and Energy-Dispersive X-ray spectroscopy (EDX) analyses, all the samples were ground with $\mathrm{SiC}$ papers down to 1000 grade. 
Final preparation was made by electropolishing $\left(6 \% \mathrm{HCl}\right.$ in ethanol, at $10-12^{\circ} \mathrm{C}$, at $25 \mathrm{~V}$ during 30s). SEM JEOL JSM 6500F equipped with EDX system (silicon diode detector Princeton Gamma tech) and a back-scattered electron (BSE) detector was used. X-ray diffraction (XRD) measurements were made on bulk, just polished samples with a Bragg-Brentano diffractometer (X'Pert Pro MRD, PANalytical, Netherlands) using a Co $\mathrm{K}_{\alpha}$ radiation with a step size of $0.08^{\circ}$ and a counting time of a few seconds per steps. The lattice parameter for each sample was calculated using the Rietveld method (MAUD software). Yet, the millimetric grain size in AC samples was at the origin of strong crystallographic texture effects and made the classical measurements of lattice parameter based on several diffraction peaks impossible. For this reason, the analysis was based on measurements limited to one high-angle $\{222\}$ peak (see 3.3 for details).

Vickers hardness tests were performed on polished samples at a load of $10 \mathrm{~kg}(980 \mathrm{~N})$. Each given value is the average value from at least ten measurements. Tensile tests were performed on threaded-head cylindrical specimens with a gauge length of $20 \mathrm{~mm}$ and a $4 \mathrm{~mm}$ diameter on an Instron 8861 testing system, with a strain rate of $10^{-3} \mathrm{~s}^{-1}$ at room temperature. For each alloy three tests were performed. Conventional yield strength value (YS) was calculated as the stress corresponding to a plastic deformation of $0.2 \%$. Strain-hardening rate (SHR) was calculated performing the first derivative of true stress over true strain: $S H R=d \sigma_{t} / d \epsilon_{t}$.

All thermodynamic simulations were performed with Thermo-Calc Software (TC2019b) using the TCHEA3 and TCFE9 databases. The calculations performed in this work led to pretty similar results from the two databases, for everything except for the description of the $\sigma$ phase. On TCFE9, $\sigma$ starts to be stable under $650^{\circ} \mathrm{C}$ whereas on TCHEA3, this phase remains stable at temperatures up to $900^{\circ} \mathrm{C}$. Precipitation of $\sigma$ at such high temperature was never observed on the Y3 grade, thus the TCFE9 database was preferred. That is why all presented results in this paper come from this database.

\section{3- Results}

\subsection{Phase stability in the $\mathrm{N}$-doped $\mathrm{Y} 3$ grade : thermodynamic prediction}

Fig. 1a shows the quasi-binary phase diagram, as a function of nitrogen content, in Y3 alloy calculated with Thermo-Calc software. The composition of studied alloys is indicated with vertical dotted lines. Following the predictions, solidification of all alloys should lead to a fully FCC structure. At lower temperatures, in solid state, the nitrogen solubility decreases and stable $\mathrm{Cr}_{2} \mathrm{~N}$ phase should precipitate: the solvus temperature of $\mathrm{Cr}_{2} \mathrm{~N}$ grows with increasing nitrogen content and is of about $890^{\circ} \mathrm{C}, 990^{\circ} \mathrm{C}$ and $1085^{\circ} \mathrm{C}$ for Y3-0.1, Y3-0.17 and Y3-0.3, respectively. Fig. $1 \mathrm{~b}$ shows in comparison, the quasi-binary $\mathrm{CoCrFeMnNi-N}$ phase diagram. The maximum solubility of nitrogen in the FCC phase is much lower than what is predicted for the $\mathrm{Y} 3$ alloy $(0.18 \mathrm{wt} . \% \mathrm{~N}$ at $1270^{\circ} \mathrm{C}$ and 0.89 wt. $\% \mathrm{~N}$ at $1300^{\circ} \mathrm{C}$ for the Cantor alloy and $\mathrm{Y} 3$ alloy respectively). Thanks to the development of High Nitrogen Stainless Steels in the past years, it is now known that the solubility of nitrogen in an austenitic matrix is strongly dependent on its composition. While $\mathrm{Cr}$ and, to a less extent, $\mathrm{Mn}$ increase the nitrogen solubility, $\mathrm{Ni}, \mathrm{Co}$, or Si have the opposite effect [32], [33], [34]. This is the reason why high nitrogen stainless steels are actually Cr-Mn rich steels [35], [36]. The presence of $20 \%$ of Co in the Cantor alloy compared to Y3 does not compensate the $5 \%$ increase in $\mathrm{Cr}$ content resulting in a much lower nitrogen solubility predicted by ThermoCalc software. It should however be noted that nitride precipitation kinetics is believed to be relatively slow compared to carbide precipitation [30] explaining why $\mathrm{Cr}_{2} \mathrm{~N}$ is nearly never observed in the as-cast state of a nitrogen-doped Cantor alloy. Klimova et al. [26] showed that for a Cantor alloy doped with 0.22 wt. $\% \mathrm{~N}\left(0.88\right.$ at. \%) there were no nitrides detected after casting whereas small $\mathrm{M}_{2} \mathrm{~N}$ particles were observed with a nitrogen content of 0.54 wt. \% $\mathrm{N}(2.2$ at. \%) which is not in agreement with the thermodynamic prediction. The question of reliability of those databases concerning nitride precipitation then arises and it could be useful to make long duration heat treatments at elevated temperatures to verify if precipitation happens as predicted. Though, we 
believe that reducing the driving force for nitride precipitation by tuning the composition of the matrix should be an efficient way to increase as much as possible the amount of nitrogen in solid solution and prevent any precipitation that could have dramatic impact on mechanical properties and toughness especially [37]. One can also note that the predicted precipitation temperature for the $\sigma$ phase decreases with the addition of nitrogen in agreement with its ability to stabilize the FCC structure. Y3 alloy has a lower FCC stability compared to the Cantor alloy (based on precipitation temperature of $\sigma$ phase, which is higher in Y3 than in Cantor alloy) due to the absence of Co. However, with the addition of nitrogen in the Y3 alloy, its FCC stability can match that of the nitrogen-free Cantor, meaning that a small amount of nitrogen can be used to avoid usage of Co for FCC stabilization.
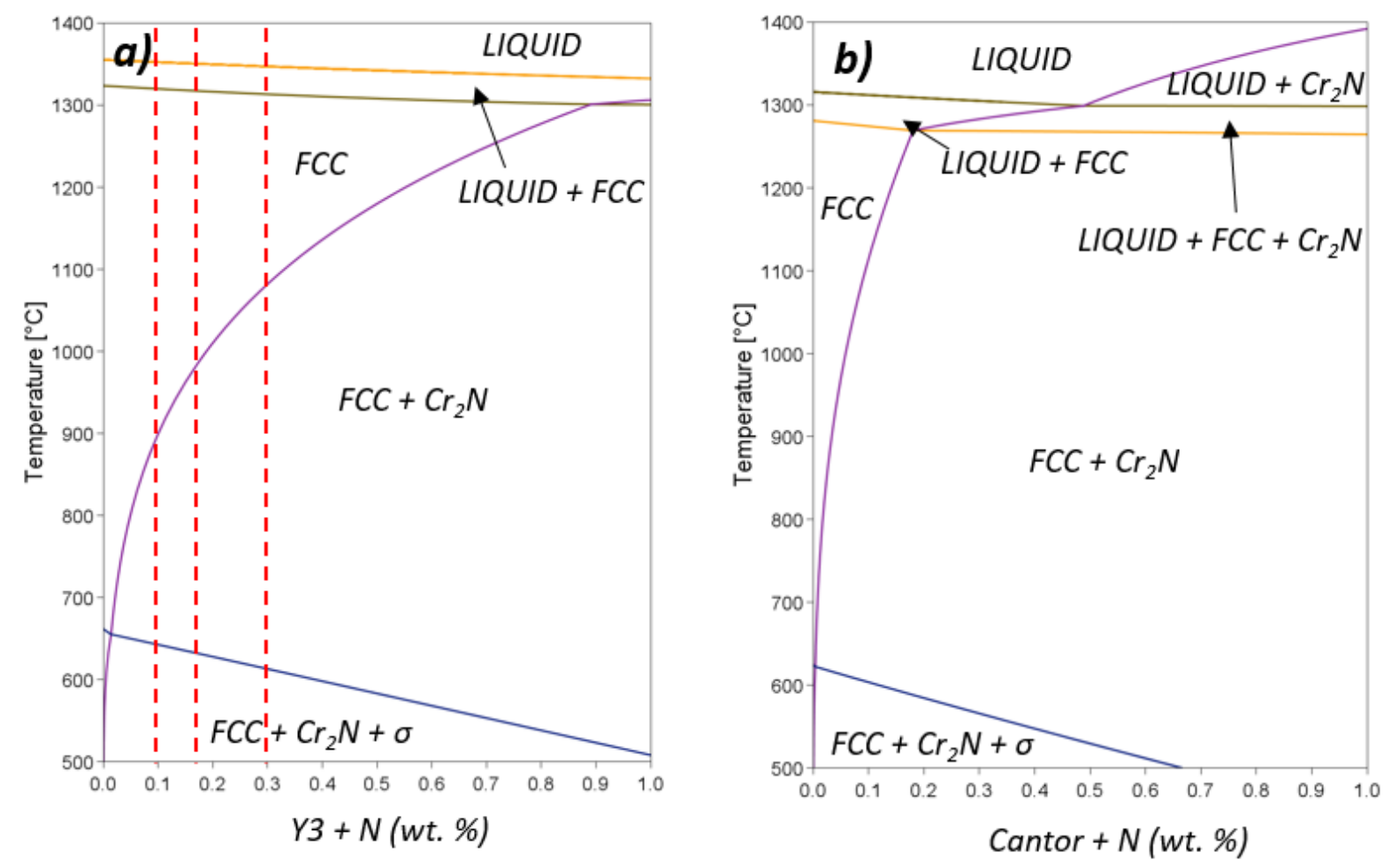

Fig. 1. Quasi-binary phase diagram of (a) Y3-N and (b) CoCrFeMnNi-N alloys calculated by Thermo-Calc software with TCFE9 database

\section{$\underline{3.2-\text { Microstructure of as-cast and forged and annealed alloys }}$}

In the AC state, a dendritic solidification structure is observed in all alloys, as shown in Fig. 2 for the Y3-0.17-AC grade. EDX maps (Fig. 3) show that in all four samples, segregation of Mn and $\mathrm{Ni}$ occurred in interdendritic regions while $\mathrm{Fe}$ and $\mathrm{Cr}$ are more concentrated into the dendrites. This observation is in agreement with previous results, as reported by Li et al. [11] and Xiang et al. [25] in the case of Cantor alloy. Comparison between Y3-0-AC and Y3-0.3-AC alloys shows that nitrogen has no significant effect on primary segregation in the studied system. These results have been confirmed by Thermo-Calc simulation using the Scheil-Gulliver model of solidification (Fig. $4 \mathrm{a}$ and $4 \mathrm{~b}$ ): it is quite clear that, as solidification proceeds, more and more $\mathrm{Ni}$ and $\mathrm{Mn}$ are rejected into the liquid while the opposite happens for $\mathrm{Fe}$ and, to a lesser extent, Cr. Interdendritic regions, which are basically the last parts of liquid to be solidified, are thus enriched in $\mathrm{Ni}$ and $\mathrm{Mn}$. The presence of nitrogen has little impact on the observed primary segregation. Finally, Fig. 4c presents the solidification path for the Y3-0.3-AC grade according to the Scheil-Gulliver model and shows that no primary precipitation should occur during solidification. This result is in agreement with the fact that, at this scale of observation, no primary precipitation was observed in any sample. This also indicates that cooling down to room temperature, which takes only a few minutes after 
solidification, is not long enough to provoke the precipitation of low temperature phases predicted at equilibrium, like $\mathrm{Cr}_{2} \mathrm{~N}$ or $\sigma$, for instance in agreement with the observations of Klimova et al. [26].

Microstructures of the forged-annealed $\left(1000^{\circ} \mathrm{C} / 2 \mathrm{~h}\right)$ samples are shown in Fig. 5 for Y3-0 and Y3-0.3. In both cases, a fully recrystallized microstructure with visible twins is observed. Similar behaviour was observed for all the other samples. Higher magnification images, focused on grain boundaries and triple junctions, (i.e. possible preferential nucleation sites for precipitates), do not show any presence of second phase even in the $\mathrm{N}$-doped grade. This is not in agreement with the thermodynamic prediction as the solvus temperature for $\mathrm{Cr}_{2} \mathrm{~N}$ precipitation in Y3-0.3 is about $1085^{\circ} \mathrm{C}$ (see 3.1). It cannot be concluded that it is due to slow precipitation kinetics, as it could also be a problem in the database's reliability. Yet, it will be assumed that this heat treatment of 2 hours at $1000^{\circ} \mathrm{C}$ is enough to cause recrystallization without excessive grain growth while keeping at least 0.3 wt. \% of nitrogen in solid solution. Finally, even if nitrogen is believed to have some effect on recrystallization and grain growth in steels [25], [38], [39], the grain size, calculated by the intercept method, does not seem to be modified with nitrogen additions $(36 \mu \mathrm{m}, 34 \mu \mathrm{m}, 28 \mu \mathrm{m}, 32 \mu \mathrm{m}$ for Y3-0, Y3-0.1, Y3-0.17 and Y3-0.3 respectively). Though, a more detailed study should be realized to properly conclude on this topic.

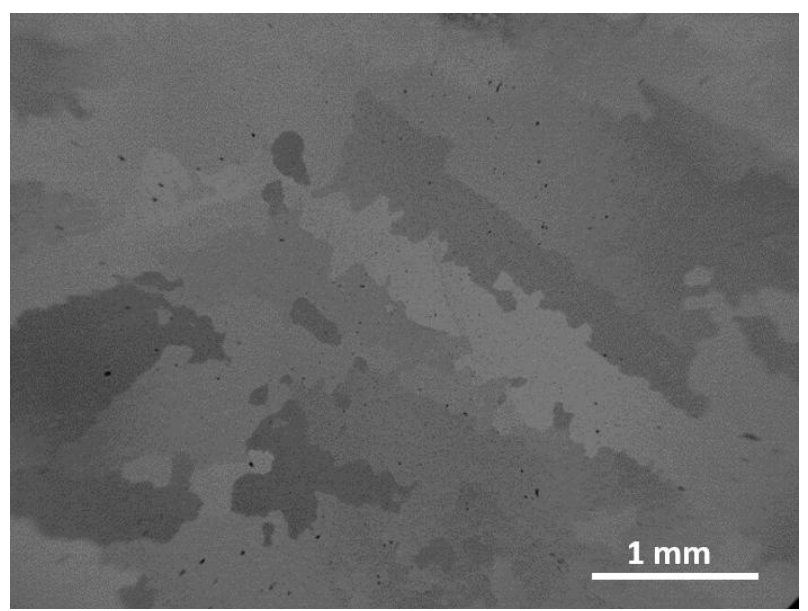

Fig. 2. SEM-BSE images of Y3-0.17-AC. Black dots are pits generated by the etching procedure. All samples present similar as-cast structure
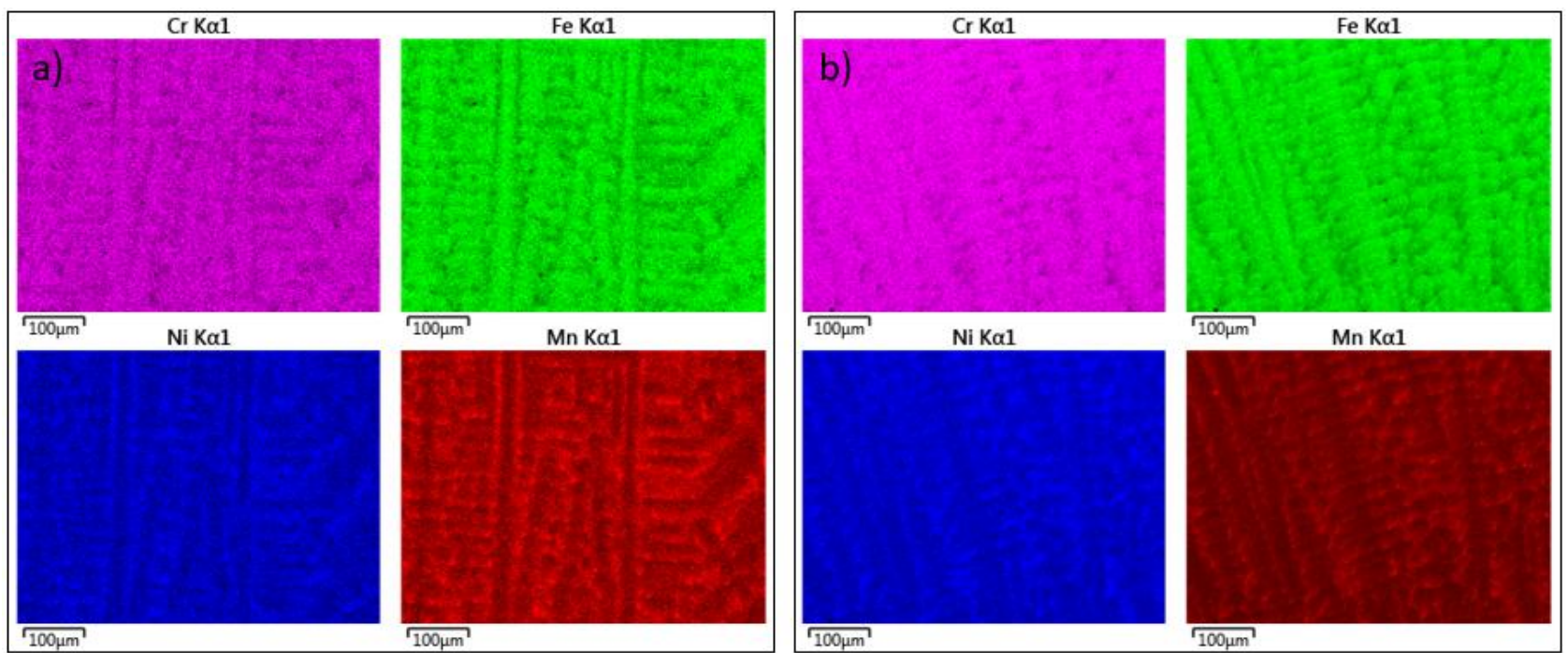

Fig. 3. EDX maps of: (a) Y3-0-AC and (b) Y3-0.3-AC 

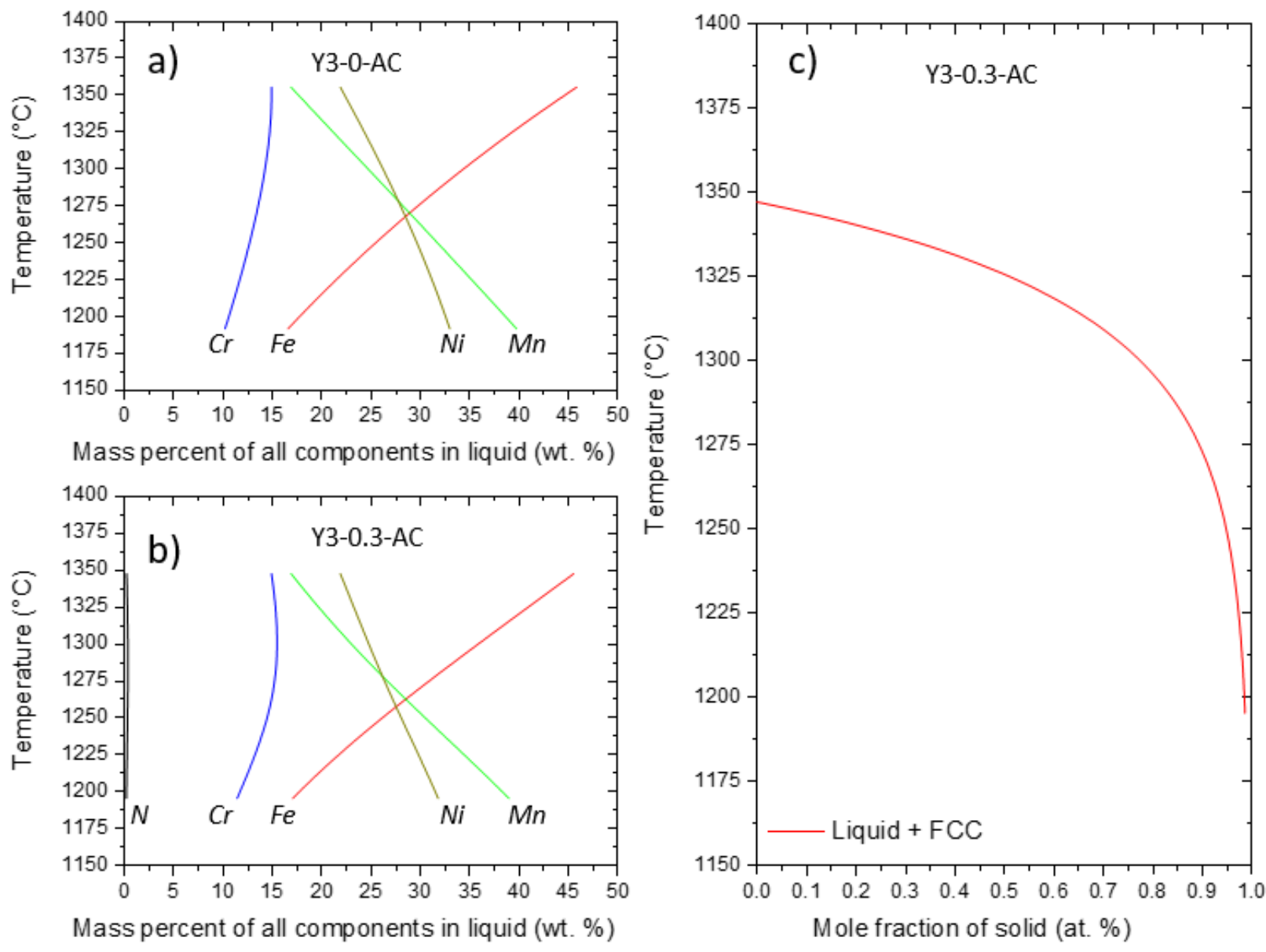

Fig. 4. (a-b) Change of chemical composition of the liquid during solidification for Y3-0-AC and Y3-0.3-AC respectively, according to the Scheil-Gulliver model; (c) Solidification path of Y3-0.3$\mathrm{AC}$ according to the Scheil-Gulliver model 


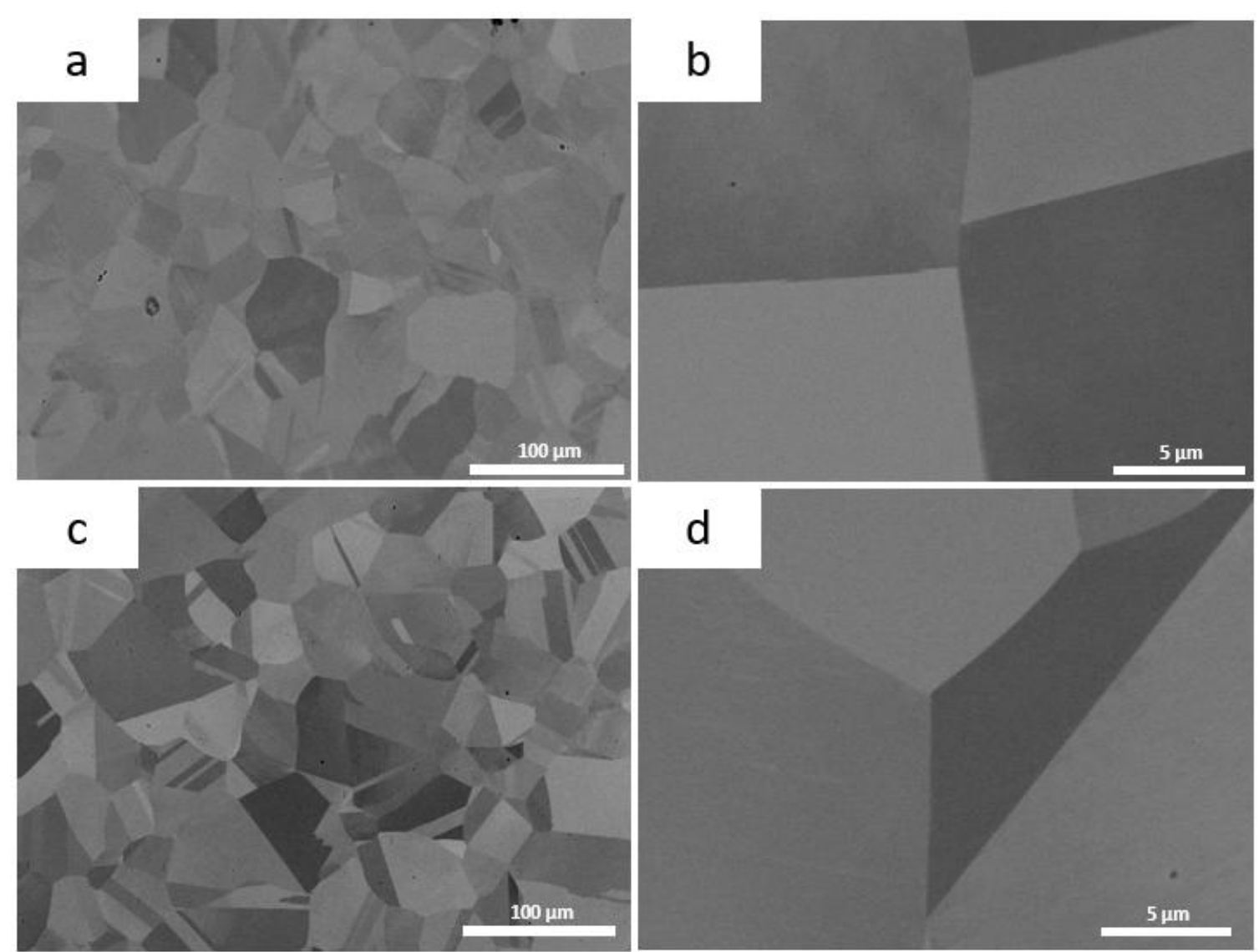

Fig. 5. SEM-BSE images of (a-b) Y3-0-FA and (c-d) Y3-0.3-FA. Black dots are pits generated by the etching procedure

\section{$\underline{3.3-\text { Effect of nitrogen on the lattice parameter value }}$}

The presence of nitrogen in the interstitial sites of the matrix should involve an expansion of the lattice parameter as observed in conventional materials or stainless steels [27], [28], [41]. XRD measurements were performed on as-cast samples to investigate this impact on the Y3 matrix. The diffractograms showed a typical FCC pattern $(\mathrm{a}=3.60 \AA$ for $\mathrm{N}$-free $\mathrm{Y} 3$ grade $)$ and no evidence of any secondary phase in all the samples, confirming that no significant precipitation occurred. Indeed, even if the presence of secondary nitrides remains possible, their volume fraction would be lower than the X-ray diffraction detectability limit (about $1 \%$ ).

As already stated, the calculation of the lattice parameter from the complete diffractogram following the standard procedures based on several peaks analyse has failed and our choice was to focus the exploitation of only one peak. To minimize errors on peak position, one can use peaks at high diffraction angles, as the experimental error decreases when the diffraction angle increases according to equation (1):

$$
\left|\Delta d_{h k l}\right|=\frac{\lambda \cos (\theta)}{2 \sin ^{2}(\theta)}|\Delta \theta|
$$

where $d_{h k l}$ is the distance between two planes with hkl indices, $\lambda$ is the radiation wavelength, and $\theta$ is the diffraction angle.

This way, we focused our effort on the peak with the highest observed diffraction angle, the $\{222\}$ peak. The area of $2 \theta$ between 116 and $121^{\circ}$ was scanned with a step of $0.08^{\circ}$. Fig. 6a shows the obtained diffraction diagrams for the four studied alloys: a shift to the lower diffraction angles as more nitrogen is added to the matrix corresponds to an increase of lattice parameter. The expansion of the lattice parameter with nitrogen addition (Fig. 6b) is linear with the amount of 
nitrogen added and corresponds to a value of $\Delta a / a_{0}(\%)=1.01 \pm 0.24 /$ wt. $\%(0.248 \pm 0.059 /$ at. $\%)$. This linear increase of lattice parameter with $\mathrm{N}$ addition is consistent with the fact that nitrogen is fully dissolved in the matrix for all as-cast studied alloys.

This value seems to be somewhat higher than usually found in the literature for stainless steels as it is generally between 0.810 /wt. \% (0.200/at. \%) and 0.977 /wt. \% (0.240 /at. \%) [16]. However, it may be dependent on composition; for instance, Ledbetter and Austin showed that the expansion for a $\mathrm{Fe}_{\text {base }} \mathrm{Mn}_{2} \mathrm{Cr}_{18} \mathrm{Ni}_{10}$ grade was 0.977 /wt. \% (0.240 /at. \%) while the value of 0.863 /wt. \% (0.212 /at. \%) was found for a $\mathrm{Fe}_{\text {base }} \mathrm{Mn}_{4} \mathrm{Cr}_{18} \mathrm{Ni}_{14}$ grade [42]. This effect might also be a medium/high entropy alloy specificity as Moravcik et al. found a value of $1.21 / \mathrm{wt}$. \% (0.255/at. \%) for the expansion of a CoCrNi matrix with carbon addition [43] and 1.01 /wt. \% (0.249 /at. \%) with nitrogen addition [24]. Finally, both Wang et al. and Klimova et al. reported very strong expansion of respectively 3.43 / wt. \% (0.78 / at. \%) on a modified FeNiMnAlCr alloy with carbon additions [12] and $2.24 /$ wt. \% (0.56/ at. \%) in a nitrogen-doped Cantor alloy [26]. However, may there be an actual difference between steels and HEAs, the reasons why this expansion would be higher in a concentrated matrix than in conventional steels are not fully understood yet.
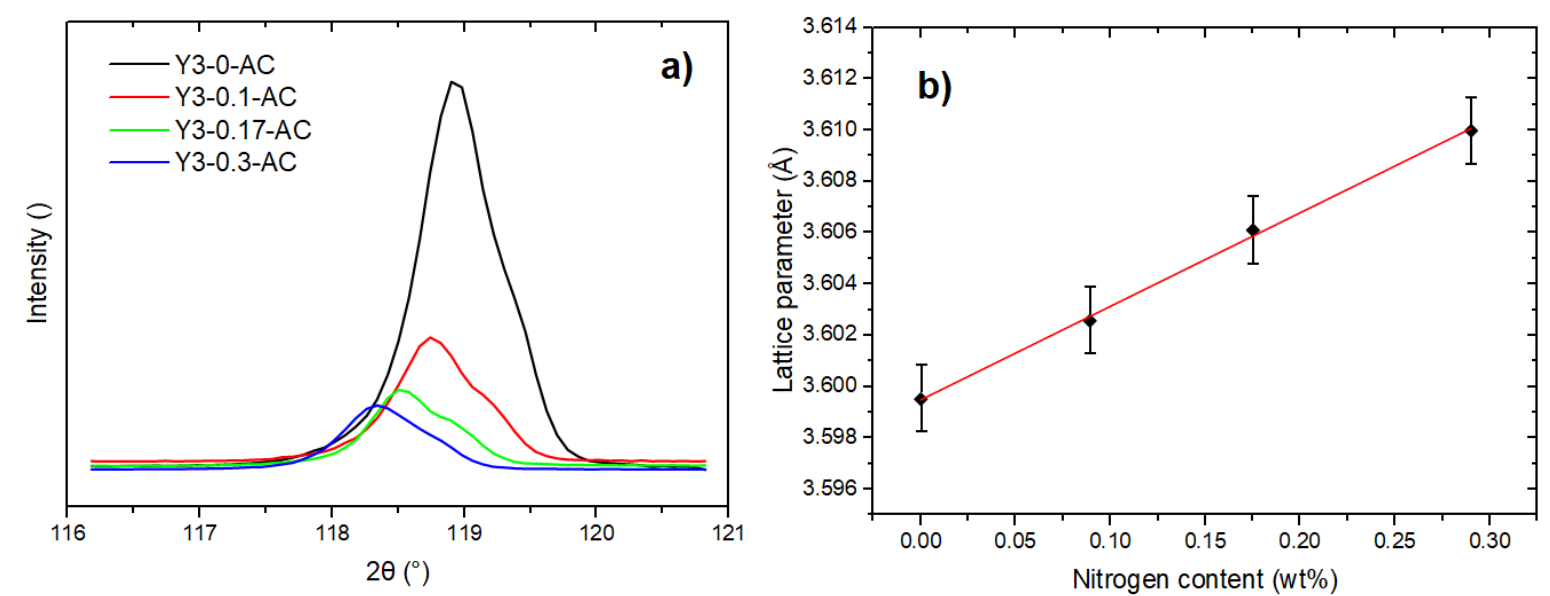

Fig. 6. (a) XRD diffractograms : superposed $\{222\}$ peaks of all the samples; (b) Evolution of the lattice parameter in the as-cast state with the amount of nitrogen added, based on the $\{222\}$ peak exploitation

\subsection{Effect of nitrogen on mechanical properties}

Tensile tests have been performed on samples in the FA state. The resulting true stress-strain curves are shown in Fig. 7a and Table 2 gives a summary of the measured mechanical properties. While the nitrogen-free Y3 alloy has quite low mechanical properties, nitrogen-doped Y3 alloy have their mechanical resistance improved without any significant impact on ductility: for example, thanks to the addition of $0.3 \mathrm{wt}$. \% of $\mathrm{N}$, the yield strength (YS) is increased by $114 \mathrm{MPa}$ and the ultimate tensile strength (UTS) by $213 \mathrm{MPa}$ while same level of ductility is observed (around 57\% elongation to fracture). The corresponding strain-hardening rates (SHR) are presented in Fig. $7 \mathrm{~b}$. While the nitrogen-free Y3 presents a monotonous decrease of its SHR value with deformation, same trend is not observed in nitrogen-doped alloys as some curvature changes occur during the tensile test. When more nitrogen is added, the change of slope becomes more important (i.e. less negative): Y3-0.17-FA has a nearly constant SHR between 6 and 12.5\% true strain while there is an increase of the SHR for the Y3-0.3-FA alloy between those two strains (from $2250 \mathrm{MPa}$ to 2340 $\mathrm{MPa}$ ). When comparing the SHR values between Y3-0-FA and Y3-0.3-FA there is a $16 \%$ increase at $6 \%$ true strain and nearly $41 \%$ increase at $12.5 \%$, which explains the higher increase of UTS than YS with nitrogen addition. However, for true strain values higher than $20 \%$, the decrease of SHR in the nitrogen-doped Y3 becomes faster and all the samples end with nearly the same SHR resulting in similar ductility. 

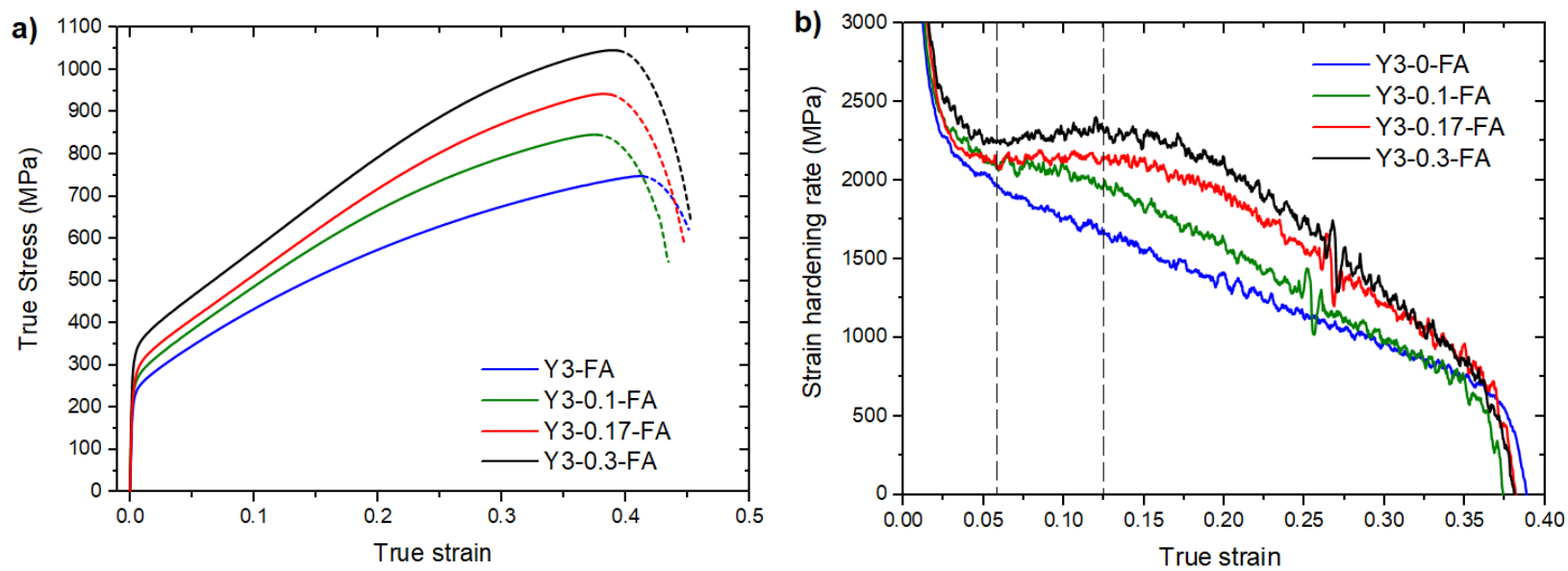

Fig. 7. (a) Evolution of room temperature true stress-strain curves of forged-annealed (FA) samples. Room temperature tensile tests with a strain rate of $10^{-3} \mathrm{~s}^{-1}$. Dashed lines represent the stress after apparition of necking as it is not a "true" stress anymore; (b) Corresponding strain-hardening rate as a function of true strain. Vertical dashed lines corresponds to respectively 6 and $12.5 \%$ true strain and represents borders of each stage of deformation.

Table 2. Mechanical properties of studied alloys in FA state. Room temperature tensile tests; strain rate of $10^{-3} \mathrm{~s}^{-1}$. Taken from the engineering stress-strain curves (not shown here). The mean value over three tests is given and the error is the standard error calculated from the tests

\begin{tabular}{cccc}
\hline Alloy & $\begin{array}{c}\text { YS } \\
\text { (MPa) }\end{array}$ & $\begin{array}{c}\text { UTS } \\
\text { (MPa) }\end{array}$ & $\begin{array}{c}\text { Elongation to fracture } \\
\text { (\%) }\end{array}$ \\
\hline Y3-0-FA & $210 \pm 10$ & $507 \pm 6$ & $58 \pm 1$ \\
\hline Y3-0.1-FA & $250 \pm 6$ & $596 \pm 6$ & $54 \pm 1$ \\
\hline Y3-0.17-FA & $272 \pm 8$ & $648 \pm 7$ & $58 \pm 1$ \\
\hline Y3-0.3-FA & $324 \pm 1$ & $720 \pm 1$ & $57 \pm 1$ \\
\hline
\end{tabular}

\section{Discussion}

\subsection{Influence of nitrogen on the yield strength}

The increase of yield strength by nitrogen addition in FA samples was calculated to be around $430 \mathrm{MPa} / \mathrm{wt}$. \% N. This value is however not solely due to the solid solution strengthening by nitrogen but rather a combination of several hardening mechanisms in addition to this one, like strain-hardening or Hall-Petch strengthening. Fig. 8 presents the results of hardness measurements with nitrogen addition in both $\mathrm{AC}$ and FA states. In the two states, a linear relationship between hardness and the nitrogen content in the matrix is measured. However the slope of the line is not the same, the hardening power of nitrogen is higher in FA samples than in AC ones.

In the AC state, an increase of $134 \mathrm{HV} / \mathrm{wt} . \% \mathrm{~N}(33 \mathrm{HV} /$ at. \% N) was measured. In principle, both solid solution hardening and precipitation hardening should be considered to explain the origin of this behaviour. The other possible factors, i.e. the grain size effect and dislocation hardening, can be neglected in this case, as the as-cast state leads to rather coarse grains and should provide a very low dislocation density. The hypothesis of the presence of nano-nitrides in at least a part of Y3-N alloys (rich in nitrogen), that would precipitate during cooling, cannot be excluded, as the performed microstructural observations were limited to a SEM scale. Yet, the presence of nanoprecipitates would hinder dislocation movement and strengthen the alloy resulting in a change of slope in the curve of hardness vs nitrogen content, which is not observed. Besides, the linear relationship strongly suggests that even in the case of the highest nitrogen content (Y3-0.3), this element is fully contained in solid solution. Additionally, section 3.3 presented another result that reinforces the hypothesis that all the nitrogen is dissolved into the matrix: a linear increase of the lattice expansion with nitrogen addition was observed for all AC alloys. Finally, if a Cantor alloy 
containing $0.22 \mathrm{wt} . \% \mathrm{~N}$ is totally free of nitrides in its as-cast state [26], our as-cast samples should have similar behaviour as the driving force for precipitation is lower than in the Cantor alloy (see section 3.1). One can thus conclude that this increase is solely due to solid solution strengthening.

In the FA state, the increase of hardness with nitrogen addition is about $210 \mathrm{HV} / \mathrm{wt}$. \% (52 $\mathrm{HV} /$ at. \%) which represents a $60 \%$ increase as compared to the as-cast state. It is reminded that no precipitation was found in the samples at the SEM scale (see Fig. 5c-d) and is then probably not responsible for this increase. However, unlike in the as-cast state, the grains are much finer and the contribution of the Hall-Petch hardening on the overall hardness is increased. While it should not be a concern because all the FA samples showed similar grain sizes (see 3.2) the strengthening by grain boundaries (modelled by the $\mathrm{K}$ parameter in the Hall-Petch relation) is believed to be increased by nitrogen addition. This should result in an increment of hardness even if grain size is kept constant. Gavriljuk et al. showed that the $\mathrm{K}$ parameter of the Hall-Petch equation was multiplied by nearly 2.8 by adding 0.54 wt. $\% \mathrm{~N}$ on a $\mathrm{Cr}_{18} \mathrm{Ni}_{16} \mathrm{Mn}_{10}$ (wt. \%) steel [44]. Similarly, Werner showed that the increase of $\mathrm{K}$ was linear with nitrogen addition (around $1140 \mathrm{MPa}^{\mathrm{m}} \mathrm{m}^{1 / 2}$ / wt. \% N) [45]. Such behaviour was also evidenced in a CoCrNi medium entropy alloy with nitrogen addition [24]. Grain refining in nitrogen alloyed HEA can thus be very interesting for additional strengthening and might explain the observed difference between the two states.

Another possible explanation for this result comes from the fact that dislocation hardening is affected by nitrogen addition. Indeed, similar behaviour was observed on an $18 \% \mathrm{Cr}-9 \% \mathrm{Ni}$ stainless steel where the increment of hardness with nitrogen addition was higher in a deformed sample than in a recrystallized one [46]. This was explained by a different dislocation density between the two states resulting in the combination of solid solution and additional hardening coming from the interaction with dislocations in the deformed state. In our case, that would indicate that the dislocation density in the FA alloy is kept high even after the heat treatment, compared to the ascast state. Such observations were already reported during primary characterizations of the Y3 alloy but the study was not pushed further [31]. Still, a difference in dislocation density between the two states coupled with nitrogen interaction with dislocations might explain such results. Nevertheless, further investigations need to be carried out to dissociate each strengthening component and its impact on the final mechanical properties.

\subsection{Influence of nitrogen on plastic deformation}

The increment of UTS with nitrogen addition is calculated to be around $760 \mathrm{MPa} / \mathrm{wt} . \% \mathrm{~N}$ which is higher than what is observed for YS increase in the same alloys (430 MPa / wt. \%). This result is linked to the modification of the strain-hardening behaviour of the Y3 alloy when nitrogen is dissolved in the matrix. Two major comments may be brought to attempt an explanation of this observation.

Firstly, bibliographic data suggest that nitrogen addition in austenitic stainless steels modifies the behaviour of dislocation by promoting planar slip [16], [44]. Thus, cross-slip is limited, affecting dislocation interactions and, ultimately, increasing work-hardening [47], [48]. Some authors claim that planar slip is promoted because nitrogen decreases the stacking fault energy (SFE). Yet, the effect of nitrogen on the SFE remains a topic of debate and depends on the alloy's composition. For example, increasing nitrogen content in a $\mathrm{Cr}_{15} \mathrm{Mn}_{17}$ steel reduced the SFE and led to a pronounced twinning during deformation [16]. However, the same authors show that in a $\mathrm{Cr}_{18} \mathrm{Ni}_{16} \mathrm{Mn}_{10}$ steel, nitrogen decreased the dissociation distance of dislocations [16], indicating that the SFE increased. The other assumption commonly encountered in literature to explain planar slip promotion is the presence of a short range order (SRO); however, this hypothesis still needs to be experimentally verified. Moreover, similar questions are considered about the impact of carbon on the SFE. Some authors [49] showed that carbon also promotes planar glide regardless of the SFE value, through an SRO effect. It seems possible that nitrogen might have a similar effect to that of carbon. 
Secondly, interesting information may be found in the SHR measurements in tensile-tested Y3 alloys, both N-free and N-doped, as represented in Fig.7b. Nitrogen addition in the Y3 alloy changes the SHR curves by inducing changes of curvature during deformation which can then be divided into three stages: stage 1 up to $6 \%$ true strain ; stage 2 between $6 \%$ true strain and about $12.5 \%$ true strain, and stage 3 starting at $12.5 \%$ until failure. A strong similarity of observed features with what is commonly observed in TWIP steels [50] is to be stressed. In such alloys, the first stage corresponds to the dislocation motion without any deformation twin. Then the second stage begins with the onset of twinning in favourably oriented grains, which counters the decrease of strain hardening. Finally, stage three is characterised by a diminishing of SHR due the reduction in the rate of twin formation, related to a twin-induced grain size decrease. It is spectacular in the present study to observe that the more nitrogen is dissolved in the matrix, the more efficient is the counter effect of twinning: starting from $0.17 \mathrm{wt} . \% \mathrm{~N}$, a stabilisation of the SHR is measured in "stage 2". This observation brings some arguments to the hypothesis that nitrogen indeed reduces the SFE in the alloy, thus facilitating the twin nucleation and so, activating twinning as an alternate deformation mode. It should be reminded that deformation twinning was already reported in the literature for $\mathrm{CoCrNi}$ or $\mathrm{CoCrFeMnNi}$ as a mechanism of their deformation at room (but also cryogenic) temperatures [25], [43] due to their relatively low SFE ( $22 \mathrm{~mJ}^{-2}{ }^{-2}$ [51] and 18-30 $\mathrm{mJ} . \mathrm{m}^{-2}$ [52], respectively). However, the monotonous decrease of the SHR of the nitrogen-free Y3 seems to indicate that no twining occurs in this alloy.

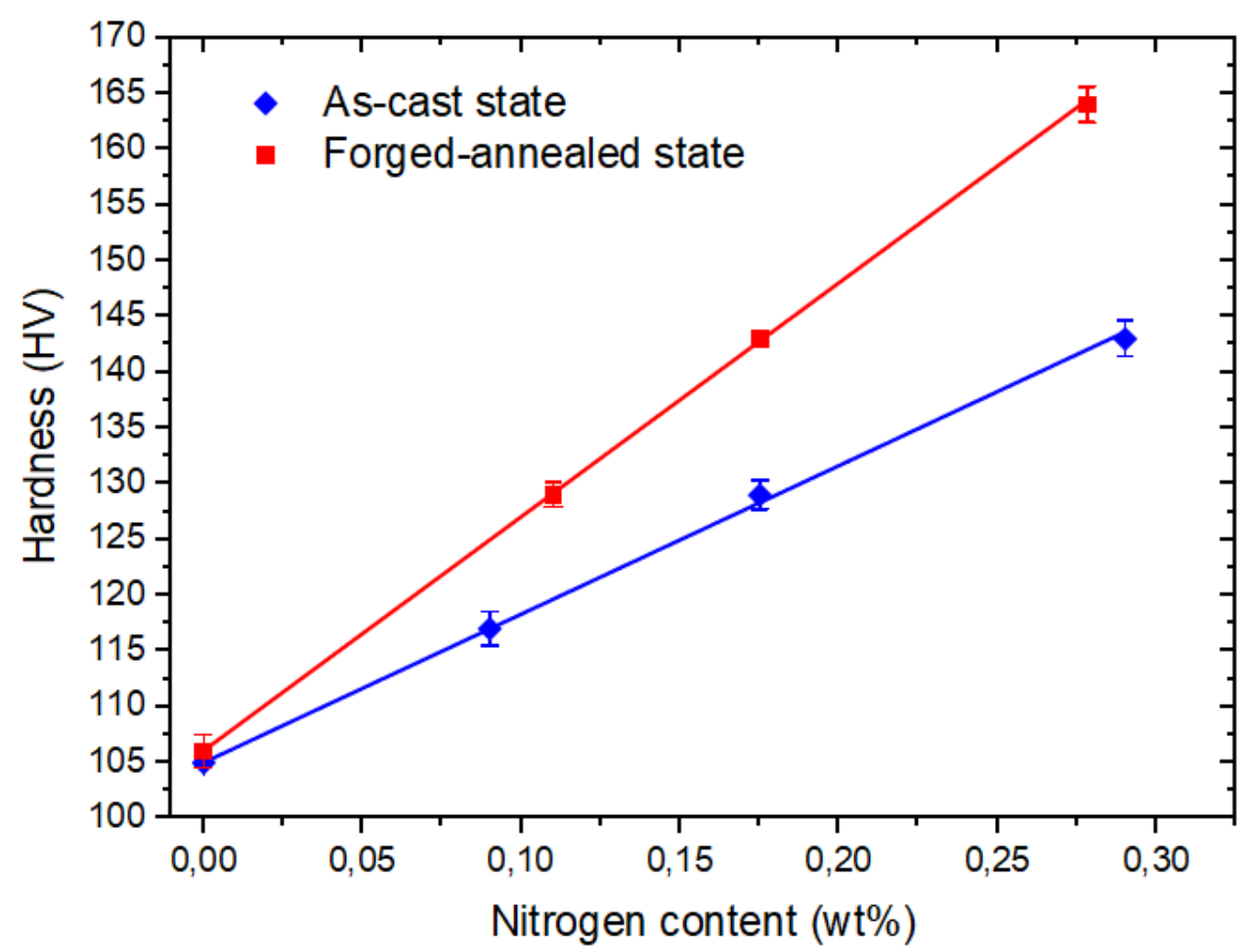

Fig. 8. Evolution of the Vickers Hardness (HV) (10 kg load) with the amount of nitrogen in the ascast and forged-annealed states

\section{5-Conclusions}

1. The effect of nitrogen was studied in an austenitic HEA, so called $\mathrm{Y} 3$ grade $\left(\mathrm{Cr}_{15} \mathrm{Fe}_{46} \mathrm{Mn}_{17} \mathrm{Ni}_{22}\right.$, at. \%). The studied alloys have been prepared by cold crucible melting, and doped with nitrogen upon casting under controlled atmosphere, with contents up to $0.3 \mathrm{wt}$. \% N. Obtained results have shown a good reproducibility and mastering of elaboration of $\mathrm{N}$-doped HEAs. In the as-cast state, 
up to $0.3 \mathrm{wt}$. \% nitrogen can be dissolved in the Y3 matrix. No precipitation occurred during natural cooling after casting.

2. The presence of nitrogen in solid solution increases the lattice parameter of the austenitic matrix; a relative increase of $\left(\Delta a / a_{0}\right) /(1 / w t \%) * 100=1.01 \pm 0.24 /$ wt. $\%(0.248 \pm 0.059 /$ at. $\%)$ was measured. This value seems slightly higher than what is currently observed in austenitic steels (bibliographic data indicate values between 0.810 and $0.977 /$ wt. \%), but similar to that reported in ternary equimolar $\mathrm{CoCrNi}$ alloy (1.03 and $1.01 / \mathrm{wt}$. \% with carbon and nitrogen addition respectively), although the level of uncertainty in measurements should be taken into account in this conclusion.

3. The evolution of hardness with nitrogen addition in the as-cast state revealed that nitrogen increases the solid solution strengthening by $134 \mathrm{HV} / \mathrm{wt} . \% \mathrm{~N}$ which is similar to what is found for conventional stainless steels. In the forged and annealed state, however, the increase of hardness with nitrogen addition is more pronounced $(210 \mathrm{HV} / \mathrm{wt} . \% \mathrm{~N})$ and might be related to the presence of several hardening mechanisms.

4. Tensile tests on forged and annealed samples showed that nitrogen indeed increases the yield strength but has an even more important effect on the ultimate tensile stress due to the modification of the strain-hardening behaviour of the alloy. Additionally, no significant effect of nitrogen on ductility has been found.

5. Therefore, nitrogen seems to be an efficient and promising alloying element to strengthen the HEA matrix while stabilizing a fully austenitic structure and high ductility.

\section{Acknowledgments}

The project is funded by the Region Auvergne-Rhône-Alpes (convention $\mathrm{N}^{\circ} 1900995901$ - 22254). The Y3 grade was designed and developed recently in collaboration with Aperam company [31]. The authors acknowledge the help of Claude Varillon (alloys elaboration) and Gilles Blanc (metallographic preparation of the alloys).

\section{Declaration of Competing Interest}

The authors declare that they have no known competing financial interests or personal relationships that could have appeared to influence the work reported in this paper.

\section{$\underline{\text { References }}$}

[1] B. Cantor, I.T.H. Chang, P. Knight, A.J.B. Vincent, Microstructural development in equiatomic multicomponent alloys, Mater. Sci. Eng. A. 375-377 (2004) 213-218. https://doi.org/10.1016/j.msea.2003.10.257.

[2] J.-W. Yeh, Recent progress in high-entropy alloys, Ann. Chim. Sci. Matér. 31 (2006) 633648. https://doi.org/10.3166/acsm.31.633-648.

[3] D.B. Miracle, O.N. Senkov, A critical review of high entropy alloys and related concepts, Acta Mater. 122 (2017) 448-511. https://doi.org/10.1016/j.actamat.2016.08.081.

[4] B. Gludovatz, A. Hohenwarter, K.V.S. Thurston, H. Bei, Z. Wu, E.P. George, R.O. Ritchie, Exceptional damage-tolerance of a medium-entropy alloy $\mathrm{CrCoNi}$ at cryogenic temperatures, Nat. Commun. 7 (2016) 10602. https://doi.org/10.1038/ncomms10602. 
[5] Y. Wang, B. Liu, K. Yan, M. Wang, S. Kabra, Y.-L. Chiu, D. Dye, P.D. Lee, Y. Liu, B. Cai, Probing deformation mechanisms of a FeCoCrNi high-entropy alloy at 293 and $77 \mathrm{~K}$ using in situ neutron diffraction, Acta Mater. 154 (2018) 79-89.

https://doi.org/10.1016/j.actamat.2018.05.013.

[6] S. Huang, W. Li, S. Lu, F. Tian, J. Shen, E. Holmström, L. Vitos, Temperature dependent stacking fault energy of FeCrCoNiMn high entropy alloy, Scr. Mater. 108 (2015) 44-47. https://doi.org/10.1016/j.scriptamat.2015.05.041.

[7] S. Gorsse, J.-P. Couzinié, D.B. Miracle, From high-entropy alloys to complex concentrated alloys, Comptes Rendus Phys. 19 (2018) 721-736. https://doi.org/10.1016/j.crhy.2018.09.004.

[8] W.H. Liu, Z.P. Lu, J.Y. He, J.H. Luan, Z.J. Wang, B. Liu, Y. Liu, M.W. Chen, C.T. Liu, Ductile CoCrFeNiMox high entropy alloys strengthened by hard intermetallic phases, Acta Mater. 116 (2016) 332-342. https://doi.org/10.1016/j.actamat.2016.06.063.

[9] J.Y. He, H. Wang, H.L. Huang, X.D. Xu, M.W. Chen, Y. Wu, X.J. Liu, T.G. Nieh, K. An, Z.P. Lu, A precipitation-hardened high-entropy alloy with outstanding tensile properties, Acta Mater. 102 (2016) 187-196. https://doi.org/10.1016/j.actamat.2015.08.076.

[10] S.-T. Chen, W.-Y. Tang, Y.-F. Kuo, S.-Y. Chen, C.-H. Tsau, T.-T. Shun, J.-W. Yeh, Microstructure and properties of age-hardenable AlxCrFe1.5MnNi0.5 alloys, Mater. Sci. Eng. A. 527 (2010) 5818-5825. https://doi.org/10.1016/j.msea.2010.05.052.

[11] Z. Li, Interstitial equiatomic CoCrFeMnNi high-entropy alloys: carbon content, microstructure, and compositional homogeneity effects on deformation behavior, Acta Mater. 164 (2019) 400-412. https://doi.org/10.1016/j.actamat.2018.10.050.

[12] Z. Wang, I. Baker, Z. Cai, S. Chen, J.D. Poplawsky, W. Guo, The effect of interstitial carbon on the mechanical properties and dislocation substructure evolution in Fe40.4Ni11.3Mn34.8A17.5Cr6 high entropy alloys, Acta Mater. 120 (2016) 228-239. https://doi.org/10.1016/j.actamat.2016.08.072.

[13] Z. Li, K.G. Pradeep, Y. Deng, D. Raabe, C.C. Tasan, Metastable high-entropy dual-phase alloys overcome the strength-ductility trade-off, Nature. 534 (2016) 227-230. https://doi.org/10.1038/nature17981.

[14] K.H. Lo, C.H. Shek, J.K.L. Lai, Recent developments in stainless steels, Mater. Sci. Eng. R Rep. 65 (2009) 39-104. https://doi.org/10.1016/j.mser.2009.03.001.

[15] J. Simmons, Strain hardening and plastic flow properties of nitrogen-alloyed Fe-17Cr-(810)Mn-5Ni austenitic stainless steels, Acta Mater. 45 (1997) 2467-2475. https://doi.org/10.1016/S1359-6454(96)00343-6.

[16] V.G. Gavriliuk, H. Berns, High nitrogen steels: structure, properties, manufacture, applications, Springer, New York, 1999.

[17] C. Sha, Z. Zhou, Z. Xie, P. Munroe, FeMnNiCoCr-based high entropy alloy coatings: Effect of nitrogen additions on microstructural development, mechanical properties and tribological performance, Appl. Surf. Sci. 507 (2020) 145101. https://doi.org/10.1016/j.apsusc.2019.145101.

[18] F. Meng, I. Baker, Nitriding of a high entropy FeNiMnAlCr alloy, J. Alloys Compd. 645 (2015) 376-381. https://doi.org/10.1016/j.jallcom.2015.05.021.

[19] W.-Y. Tang, M.-H. Chuang, H.-Y. Chen, J.-W. Yeh, Microstructure and mechanical performance of new Al0.5CrFe1.5MnNi0.5 high-entropy alloys improved by plasma nitriding, Surf. Coat. Technol. 204 (2010) 3118-3124. https://doi.org/10.1016/j.surfcoat.2010.02.045.

[20] D.E. Jodi, N. Choi, J. Park, N. Park, Mechanical Performance Improvement by Nitrogen Addition in N-CoCrNi Compositionally Complex Alloys, Metall. Mater. Trans. A. (2020). https://doi.org/10.1007/s11661-020-05738-9.

[21] D.E. Jodi, J. Park, N. Park, Precipitate behavior in nitrogen-containing CoCrNi mediumentropy alloys, Mater. Charact. 157 (2019) 109888. https://doi.org/10.1016/j.matchar.2019.109888.

[22] D.E. Jodi, J. Park, N. Park, Strengthening of ultrafine-grained equiatomic CoCrFeMnNi highentropy alloy by nitrogen addition, Mater. Lett. 258 (2020) 126772. https://doi.org/10.1016/j.matlet.2019.126772. 
[23] Y. Han, H. Li, H. Feng, K. Li, Y. Tian, Z. Jiang, Enhancing the strength and ductility of CoCrFeMnNi high-entropy alloy by nitrogen addition, Mater. Sci. Eng. A. (2020) 139587. https://doi.org/10.1016/j.msea.2020.139587.

[24] I. Moravcik, H. Hadraba, L. Li, I. Dlouhy, D. Raabe, Z. Li, Yield strength increase of a CoCrNi medium entropy alloy by interstitial nitrogen doping at maintained ductility, Scr. Mater. 178 (2020) 391-397. https://doi.org/10.1016/j.scriptamat.2019.12.007.

[25] F. Xiong, R. Fu, Y. Li, D. Sang, Effects of nitrogen alloying and friction stir processing on the microstructures and mechanical properties of $\mathrm{CoCrFeMnNi} \mathrm{high-entropy} \mathrm{alloys,} \mathrm{J.} \mathrm{Alloys}$ Compd. 822 (2020) 153512. https://doi.org/10.1016/j.jallcom.2019.153512.

[26] M. Klimova, D. Shaysultanov, A. Semenyuk, S. Zherebtsov, G. Salishchev, N. Stepanov, Effect of nitrogen on mechanical properties of $\mathrm{CoCrFeMnNi}$ high entropy alloy at room and cryogenic temperatures, J. Alloys Compd. 849 (2020) 156633. https://doi.org/10.1016/j.jallcom.2020.156633.

[27] F. Xiong, R. Fu, Y. Li, B. Xu, X. Qi, Influences of nitrogen alloying on microstructural evolution and tensile properties of CoCrFeMnNi high-entropy alloy treated by cold-rolling and subsequent annealing, Mater. Sci. Eng. A. 787 (2020) 139472. https://doi.org/10.1016/j.msea.2020.139472.

[28] Y. Xie, H. Cheng, Q. Tang, W. Chen, W. Chen, P. Dai, Effects of N addition on microstructure and mechanical properties of CoCrFeNiMn high entropy alloy produced by mechanical alloying and vacuum hot pressing sintering, Intermetallics. 93 (2018) 228-234. https://doi.org/10.1016/j.intermet.2017.09.013.

[29] I. Moravcik, J. Cizek, L. Gouvea, J. Cupera, I. Guban, I. Dlouhy, Nitrogen Interstitial Alloying of CoCrFeMnNi High Entropy Alloy through Reactive Powder Milling, Entropy. 21 (2019) 363. https://doi.org/10.3390/e21040363.

[30] Y.-S. Jung, S. Kang, K. Jeong, J.-G. Jung, Y.-K. Lee, The effects of N on the microstructures and tensile properties of $\mathrm{Fe}-15 \mathrm{Mn}-0.6 \mathrm{C}-2 \mathrm{Cr}-\mathrm{xN}$ twinning-induced plasticity steels, Acta Mater. 61 (2013) 6541-6548. https://doi.org/10.1016/j.actamat.2013.07.036.

[31] J. Olszewska, Conception and development of a novel grad of high resistance High Entropy Alloy from the CrFeMnNi familly, PhD Thesis, Ecole des Mines de Saint Etienne, 2019.

[32] Y. Kojima, M. Inouye, Y. Yamada, Solubility and Diffusivity of Nitrogen in Liquid IronNickel and Iron-Cobalt Alloys at $1600^{\circ} \mathrm{C}$, Trans. Iron Steel Inst. Jpn. 15 (1975) 599-605. https://doi.org/10.2355/isijinternational1966.15.599.

[33] G. Balachandran, M.L. Bhatia, N.B. Ballal, P.K. Rao, Some Theoretical Aspects on Designing Nickel Free High Nitrogen Austenitic Stainless Steels., ISIJ Int. 41 (2001) 1018-1027. https://doi.org/10.2355/isijinternational.41.1018.

[34] R.P. Reed, Nitrogen in austenitic stainless steels, JOM. 41 (1989) 16-21. https://doi.org/10.1007/BF03220991.

[35] J.C. Rawers, G. Asai, J.S. Dunning, Change in mechanical properties and microstructure of 201 stainless steel with increased nitrogen alloying, J. Mater. Res. 9 (1994) 3160-3169. https://doi.org/10.1557/JMR.1994.3160.

[36] T.-H. Lee, S.-J. Kim, S. Takaki, Time-temperature-precipitation characteristics of highnitrogen austenitic $\mathrm{Fe}-18 \mathrm{Cr}-18 \mathrm{Mn}-2 \mathrm{Mo}-0.9 \mathrm{~N}$ steel, Metall. Mater. Trans. A. 37 (2006) 3445-3454. https://doi.org/10.1007/s11661-006-1040-8.

[37] J.W. Simmons, Mechanical properties of isothermally aged high-nitrogen stainless steel, Metall. Mater. Trans. A. (n.d.) 17.

[38] D.W. Kim, Influence of nitrogen-induced grain refinement on mechanical properties of nitrogen alloyed type 316LN stainless steel, J. Nucl. Mater. 420 (2012) 473-478. https://doi.org/10.1016/j.jnucmat.2011.11.001.

[39] M. Song, R. Zhou, J. Gu, Z. Wang, S. Ni, Y. Liu, Nitrogen induced heterogeneous structures overcome strength-ductility trade-off in an additively manufactured high-entropy alloy, Appl. Mater. Today. 18 (2020) 100498. https://doi.org/10.1016/j.apmt.2019.100498. 
[40] X. Tao, A. Matthews, A. Leyland, On the Nitrogen-Induced Lattice Expansion of a Nonstainless Austenitic Steel, Invar 36®, Under Triode Plasma Nitriding, Metall. Mater. Trans. A. 51 (2020) 436-447. https://doi.org/10.1007/s11661-019-05526-0.

[41] A. Krawitz, R. Sinclair, On the lattice parameter of non-random solid solutions, Philos. Mag. J. Theor. Exp. Appl. Phys. 31 (1975) 697-712. https://doi.org/10.1080/14786437508226548.

[42] H.M. Ledbetter, M.W. Austin, Dilation of an fcc $\mathrm{Fe}-\mathrm{Cr}-\mathrm{Ni}$ alloy by interstitial carbon and nitrogen, Mater. Sci. Technol. 3 (1987) 101-104. https://doi.org/10.1179/mst.1987.3.2.101.

[43] I. Moravcik, V. Hornik, P. Minárik, L. Li, I. Dlouhy, M. Janovska, D. Raabe, Z. Li, Interstitial doping enhances the strength-ductility synergy in a CoCrNi medium entropy alloy, Mater. Sci. Eng. A. 781 (2020) 139242. https://doi.org/10.1016/j.msea.2020.139242.

[44] V.G. Gavriljuk, H. Berns, C. Escher, N.I. Glavatskaya, A. Sozinov, Yu.N. Petrov, Grain boundary strengthening in austenitic nitrogen steels, Mater. Sci. Eng. A. 271 (1999) 14-21. https://doi.org/10.1016/S0921-5093(99)00272-5.

[45] E. Werner, Solid Solution and Grain Size Hardening of Nitrogen-alloyed Austenitic Steels, (n.d.) 6.

[46] M. Tendo, Y. Tadokoro, K. Suetsugu, T. Nakazawa, Effects of Nitrogen, Niobium and Molybdenum on Strengthening of Austenitic Stainless Steel Produced by Thermo-Mechanical Control Process., ISIJ Int. 41 (2001) 262-267. https://doi.org/10.2355/isijinternational.41.262.

[47] T. Masumura, Y. Seto, T. Tsuchiyama, K. Kimura, Work-Hardening Mechanism in HighNitrogen Austenitic Stainless Steel, Mater. Trans. 61 (2020) 678-684. https://doi.org/10.2320/matertrans.H-M2020804.

[48] J.W. Simmons, Overview: high-nitrogen alloying of stainless steels, Mater. Sci. Eng. A. 207 (1996) 159-169. https://doi.org/10.1016/0921-5093(95)09991-3.

[49] J.D. Yoo, K.-T. Park, Microband-induced plasticity in a high Mn-Al-C light steel, Mater. Sci. Eng. A. 496 (2008) 417-424. https://doi.org/10.1016/j.msea.2008.05.042.

[50] B.C. De Cooman, Y. Estrin, S.K. Kim, Twinning-induced plasticity (TWIP) steels, Acta Mater. 142 (2018) 283-362. https://doi.org/10.1016/j.actamat.2017.06.046.

[51] M. Schneider, E.P. George, T.J. Manescau, T. Záležák, J. Hunfeld, A. Dlouhý, G. Eggeler, G. Laplanche, Analysis of strengthening due to grain boundaries and annealing twin boundaries in the CrCoNi medium-entropy alloy, Int. J. Plast. 124 (2020) 155-169. https://doi.org/10.1016/j.ijplas.2019.08.009.

[52] E.P. George, W.A. Curtin, C.C. Tasan, High entropy alloys: A focused review of mechanical properties and deformation mechanisms, Acta Mater. 188 (2020) 435-474. https://doi.org/10.1016/j.actamat.2019.12.015. 